\title{
Experimental Approach on a Swirl Flow and Heat Transfer Coefficient Using a 3-D Stereo-PIV and Liquid Crystals
}

\author{
Daisy Galeana ${ }^{1}$, Asfaw Beyene ${ }^{2}$ \\ ${ }^{1}$ Solar Turbines Incorporated and Joint Doctoral Program at San Diego State University \\ 2200 Pacific Highway, San Diego, USA \\ Galeana_daisy@solarturbines.com \\ ${ }^{2}$ San Diego State University \\ 5500 Campanile Dr., San Diego, USA \\ abeyene@mail.sdsu.edu
}

\begin{abstract}
The paper discusses effects on the heat transfer coefficients and velocity flow field of a circular chamber from the standpoint of internal cooling system in a gas turbine blade. The research investigates the convective heat transfer by means of Thermochromic Liquid Crystals and velocity flow field using 3-D Stereo-PIV (Particle Image Velocimetry). The application of a swirl cooling flow technique in combination with convective heat transfer measurements at three different Reynolds numbers, 7,000, 14,000, and 21,000, measure the effect of the flow on the circular chamber. The study focuses on finding the effect of swirl number with higher heat transfer coefficient created by the continuous tangential air injection that represents the gas turbine blade cooling path. An experimental test stand consists of a circular chamber system with seven discrete tangential jets created to allow measurement of the swirl flow velocity field in full range of the chamber. The circular chamber is made of clear acrylic, which permits a strong visualization of the flow characteristics using TechPlot. Circumferential velocity, axial velocity, vorticity, and kinematic energy are described from time averaged surveys of the circumferential component at 33 locations along the chamber length. The swirl numbers are given based on the flux of circumferential and axial momentum. The heat transfer coefficient and Nusselt number are calculated based on the inlet total temperature. The Nusselt numbers begin to vary near each air inlet in both axial and circumferential directions. At $\mathrm{Re}=14,000$, the experiment indicates an average Nusselt number is higher in the middle of the chamber length due to the seven air inlets.
\end{abstract}

Keywords: Swirl Flow, Heat Transfer, Turbine Blade.

\section{Introduction}

Gas turbine engines require a turbine inlet temperature (TRIT) that is much higher than the average material melting temperature, which limits the material choices of the turbine blades. Therefore, internal flow passages are an important solution to the turbine blade design to keep the TRIT within a safety limit. From all the internal blade cooling methods, swirl cooling is used to protect the turbine blades from hot gas thermal deterioration. In the swirl cooling technique, using a lengthwise continuous tangential injection is the focus of this investigation. The technique utilizes a three-dimensional swirl cooling motion generated by seven tangential air inlets introduced at 90 degrees to the circular chamber axis. Seven continuous air inlets along the chamber provides strong swirl formation, which are sustained over its entire length. The objective of the circular chamber swirl cooling flow is to enhance surface heat transfer and mass transfer at its maximum levels. To maximize the understanding of the physical mechanism and to optimize the internal cooling design, a considerable number of studies have been conducted during the past 30 years by Moon et al. [1], Glezer et al. [2], and Ligrani et al. [3] adding to the first proposed design by Kreith et al. [4]. In the early studies, experiments dealt with producing a swirl in a tube flow and found the augmentation surface heat transfer rates relative to non-swirl flows [1]-[8][13]-[16]. Early research included tangential jets from wall slots to generate large scale swirl in internal tube flow. Study by Camci et al. [5] and Chang et al. [6] indicated that the heat transfer coefficient increases when compared to previous investigations by developing a liquid crystal thermography system in the rotating environment. The study demonstrates that the use of liquid crystals thermography can be employed in steady-state heat transfer experiment with superior spatial resolution and reliable accuracy.

The focus is on the swirl cooling flow technique, how the coolant air is directed to the turbine blades to decrease temperature as it circulates through the blade's internal passages. Fig. 1 shows passages where the continuous injection 
creates a swirling flow along the turbine blade leading edge. This experiment focuses on the flow field characteristics, where the setup allows for easy capture of flow behaviour through an acrylic walled model. The continuous swirl flow is used for both swirl flow and heat transfer tests, which are maintained by continuous injection of tangential flow and generated at all seven air inlets which decay with downstream distance. The results originated from the internal swirl cooling flow by utilizing 3-D Stereo- Particle Image Velocimetry (PIV) and heat transfer studies utilizing liquid crystal technique performed using the range of Re numbers simulating the internal flow of a turbine blade. The preference of using Stereo-PIV over other flow measurement methods is the capturing of the velocity flow field and accuracy on the exact direction information of multiple particles. The heat transfer results are from a surface Nusselt number distribution on a second coated acrylic circular chamber with Thermochromic Liquid Crystal (TLC).

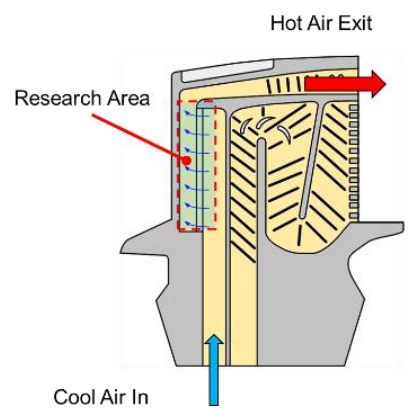

Fig. 1: Turbine Blade's Internal Cooling Flow, Glezer, et al. [7].

\section{Model Development and Experimental Apparatus}

\subsection{Model Structure}

A detailed circular chamber instrumented with standard thermocouples and pressure transducers are used for air and swirl flow measurements at chosen coordinates shown in Fig. 2. The setup consists of a circular chamber made of acrylic with seven discrete tangential air inlets designed to allow detail measurements of the flow field. For heat transfer measurements, an acrylic circular chamber is coated with TLC paint that produces a green hue for a controlled narrow temperature range. Fig. 2 also shows one end of the chamber is closed and the other end is opened to the atmosphere. The cylinder length, inner diameter, and outer diameter are $1.07 \mathrm{~m}, 0.075 \mathrm{~m}$, and $0.088 \mathrm{~m}$, respectively. The air enters the plenum and passes into a rectangular heating mesh, hot air leads to rectangular cross-sectional air inlets with individual hydraulic diameter of $0.011 \mathrm{~m}$, which are connected to the principal swirl chamber so that one surface is tangential to the chamber's inner circumference.

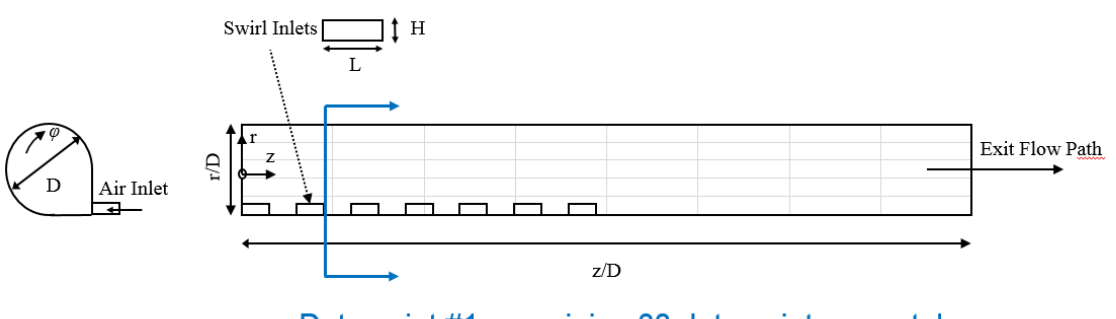

Data point \#1, remaining 33 data points were taken

Fig. 2: Swirl Chamber Schematic, Galeana et al. [8].

\subsection{Thermochromic Liquid Crystal (TLC)}

TLC are materials that change their reflected color when illuminated by white light, as a function of temperature. Videos of tests record the color change over time and the data collected is combined with temperature probe data to produce heat transfer coefficient results. The in-house software calculates the heat transfer coefficients from green time data, thermocouple temperatures, and model material properties by using a semi-infinite thermal solution. The TLC helps focus on the most desirable surface area by painting the cylindrical chamber with black and liquid crystal paints that facilitate a measurement 
accuracy to 1 micron. From Fig. 3 only the surface area optics suitable for the spatial resolution are painted. A coupon is created for calibration using the same process as the chamber, where both are coated with liquid crystal paint followed by black paint. After the full test article setup is connected, mass flow is established, the current is switched on to heat up the heating mesh, and the air leaving the heater ramps up to the required temperature, all while cameras are recording the color changes.

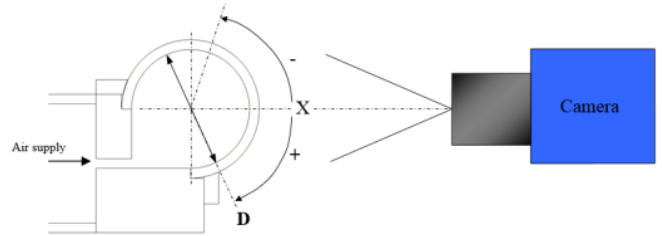

Fig. 3: Painted Surface and Camera View, Galeana et al. [8].

\subsection{Experimental Setup}

Two Sony video cameras connected to two PCs and one VXI EX1048 (VXI) temperature scanner collect data using inhouse software to synchronize the entire liquid crystal experiment. The data is collected immediately after the system is heat soaked to the targeted temperature, as shown in Fig. 4. The video file is converted to an AVI file, to import it into a Solar Turbines Program Liquid Crystal Imagine Analyzer (LCIA). The temperature of the air entering the swirl chamber is measured using fourteen calibrated thermocouples equally separated across the length of the chamber. All measurements are collected when the swirl chamber is at a steady state and heating mesh on the plenum reaches $60^{\circ} \mathrm{C}$.

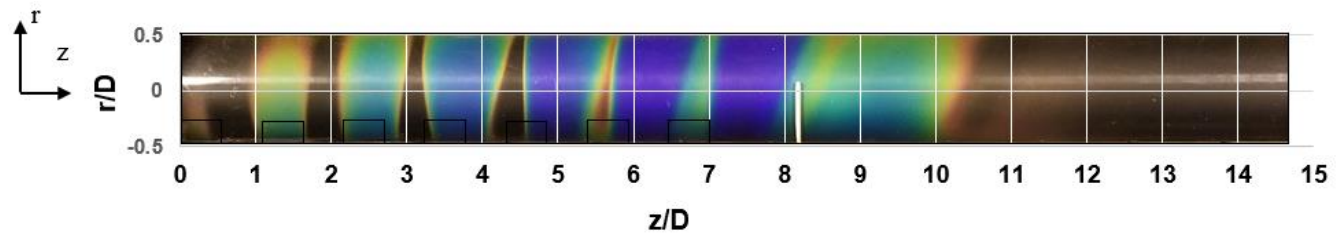

Fig. 4: Video Images as the TLC Changes Color, Galeana et al. [8].

The laboratory setup is a LaVision stereo-PIV system, which as shown in Fig. 5 includes a LaVision PC, two ImagerproX cameras, two Nd-YAG laser, and a LaVision particle seeder. The experimental study observes the air seeded with olive oil particles with diameters in the range of $1-3 \mu \mathrm{m}$ and specific gravity of 0.703 . These seeds are the tracer particles that were specifically chosen because of their size, which assures the tracer particle motion best reflects the actual flow path. The seeding particles in the fluid are scattered throughout the chamber where the laser light can be captured by the video acquisition system. Two CCD cameras with high speed and high resolution (Vision Research, Phantom v7.3. 800_600 pixels, 12 bit) seize images of the illuminated PIV particles at a rate of 100 frames per second.

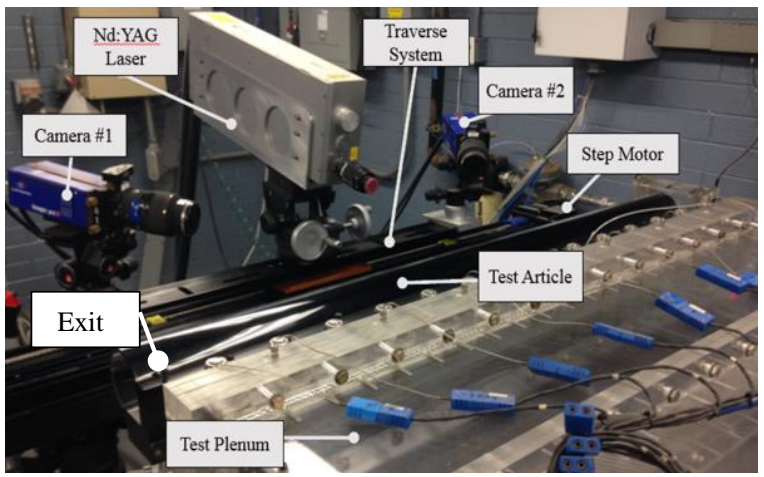

Fig. 5: Experimental Setup, Galeana et al. [8]. 


\subsection{Liquid Crystal Paint Calibration}

The coupons are painted with the SPB100 black primer paint and then coated with eight layers of the R35C1W liquid crystal paint from Liquid Crystal resources. A copper coupon plate is created with an embedded T-type thermocouple bonded on a Peltier-thermoelectric module (Peltier Bridge) heater (Tellurex C2-50-1514). The thermocouples are connected to the EX1048 followed by connections to the thermoelectric module to the power supply with a multimeter in parallel with the power supply to detect voltage. Supplying 2 Volts to the thermoelectric module while simultaneously flipping the test switch on the thermo flow bench is used to correlate the time between the EX1048 data and video. The estimated temperatures for the entire input domain of the green time image is generated from probe data and then mapped.

\subsection{Swirl Flow Using 3-D Stereo-PIV}

PIV has proven to be an effective tool for demanding conditions of flow velocity measurements, such as a turbine engine environment. The oil particles are provided by seeder (LAVision) for velocity measurements. Acrylic also works well for infrared imaging because its surface emissivity ranges from 0.60 to 0.65 [9]. Particle images are taken using two high resolution cameras and processed by DaVis software as shown in Fig. 6. Data is post-processed in DaVis, velocity calculations are conducted in MATLAB, and Techplot 360 is used for data visualization. Flow along the chamber is measured at 33 different cross-sectional locations along the length of the chamber.

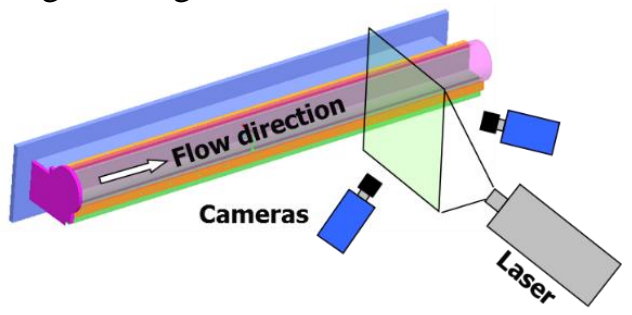

Fig. 6: Experimental Setup 3-D, Galeana et al. [10].

\subsection{Uncertainty Estimates}

Uncertainty of temperatures measured with thermocouples is $\pm 0.15^{\circ} \mathrm{C}$. The uncertainties of total pressure and static pressure (relative to atmospheric pressure) include $\pm 0.40 \%$ and $\pm 0.20 \%$ in Pascals, respectively. Uncertainty estimates are based on 95 percent confidence levels, and determined using procedures described by B. Weineke [11]. Nusselt number uncertainty is around $10 \%$. Reynolds number uncertainty is \pm 410 which is about 2.5 percent for Reynolds number equal to 14,000 .

\section{Results and Discussion}

\subsection{Swirl Number Magnitudes}

All three Re numbers (nominal and $\pm 50 \%$ ) are presented at $\operatorname{Re}=7,000,14,000$, and 21,000 and flow rate measurements were plotted for all velocity profiles involving the TLC experiment. The definition of the swirl number is the ratio of the flux of angular momentum and the flux of axial momentum per Moon, et al. [1] and Hedlund, et al. [9]. Fig. 7 shows swirling increasing at each air inlet and peaks are much higher at these locations than areas where there are no air inlets. At z/D 1 to 3 the swirl number is particularly high because of the proximity of the first two air inlets to one swirl chamber and face which restricts the axial component of the flow. Therefore, the axial flow momentum is moderately small at this location given a high swirl number along the air inlet length. The swirl number reveals a decreasing pattern as the exit is approached between $\mathrm{z} / \mathrm{D}=7$ and 15 .

$$
\mathrm{S}_{\mathrm{N}}=\frac{\int_{r=0}^{R} \rho V_{\phi} V_{z} 2 \pi r^{2} d r}{R \int_{r=0}^{R} \rho V_{z}^{2} 2 \pi r d r}
$$




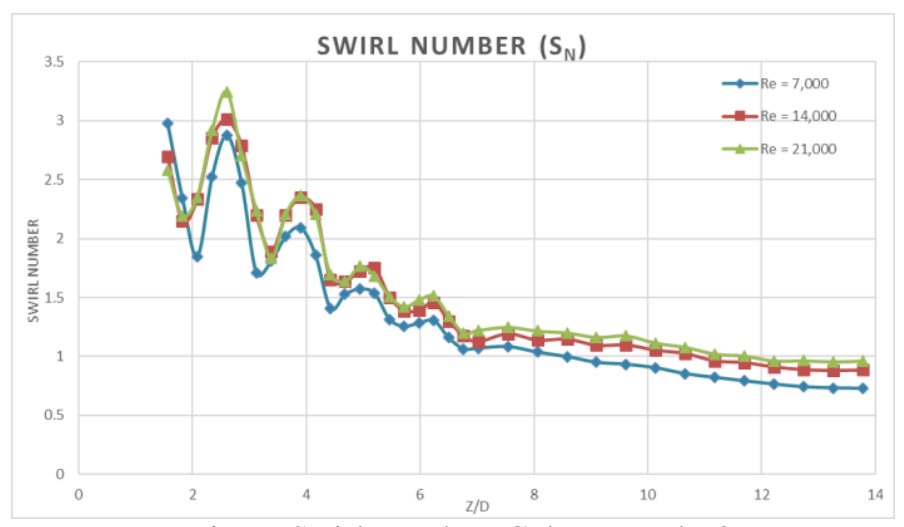

Fig. 7: Swirl Number, Galeana et al. [8].

\subsection{Flow Field Results}

Circumferential velocity, $\mathrm{V} \phi$, as shown in Fig. 8, exhibits an increase in the radial direction and later decreases near the swirl chamber wall. The $\mathrm{V} \phi$ ranges of the three Re numbers are from -15 to $15 \mathrm{~m} / \mathrm{s}$, as shown in Fig. 8(a). As the Re number increases the axial velocity, Vz, increases on the outer wall region, as shown in Fig. 8(b). The outer wall region velocity is intensifying across the length reaching the highest velocity at the second half of the swirl chamber length. Vorticity measurements show that vorticity highest reading values are closer to the wall shown in Fig. 9, due to the frictional effects. At $\operatorname{Re}=7,000$ vorticity measurements ranges from 10 to -60 , then at nominal $\operatorname{Re}=14,000$ the range increases from 25 to 125 , at $\operatorname{Re}=21,000$ vorticity measurements ranges from 50 to -200 .

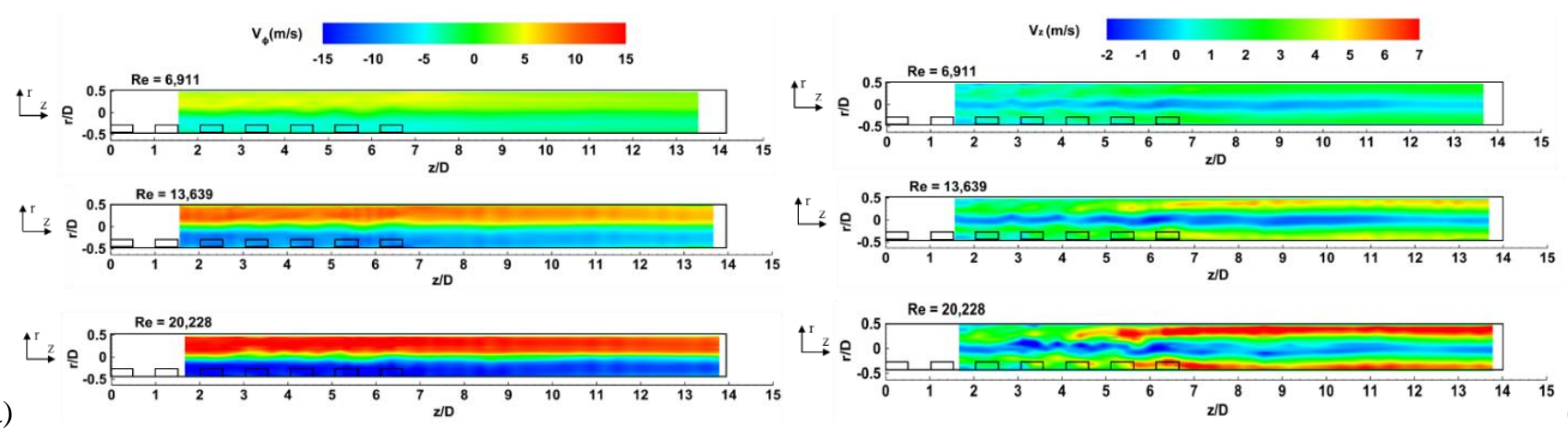

Fig. 8: (a) Circumferential and (b)Axial Velocity, Galeana et al. [10].

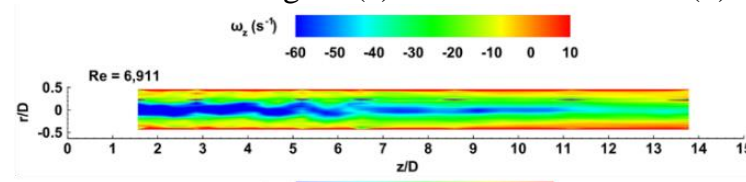

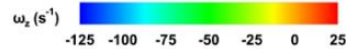
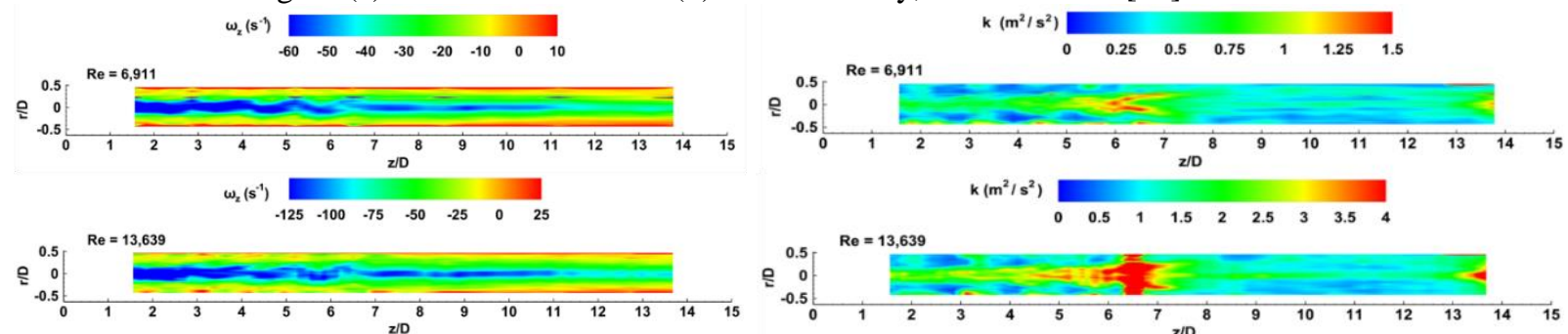

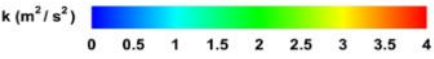

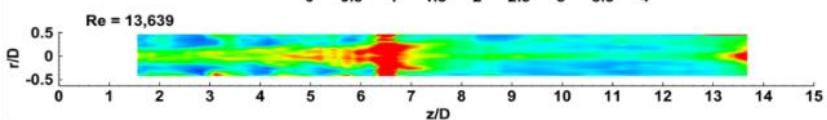

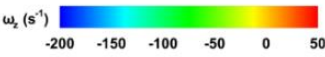

$\begin{array}{rlllllll}k\left(\mathrm{~m}^{2} / \mathrm{s}^{2}\right) & 0 & & & & & & \\ 0 & 1 & 2 & 3 & 4 & 5 & 7 & 8\end{array}$
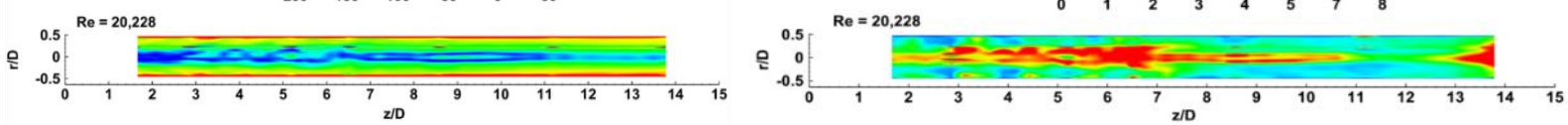

(a)

Fig. 9: (a) Vorticity and (b) Turbulent Kinetic Energy, Galeana et al. [10]. 


\subsection{Heat Transfer Coefficient (HCOEF) and Nusselt Number Magnitudes throughout the Swirl Chamber}

As the Re number increases the heat transfer coefficient largely increases due to the high swirl flow. The impact of the Re number is seen in Fig. 10, where heat transfer measurement results for this configuration were plotted against the normalized lengthwise distance. It appears that the employment of the TLC helps calculate the heat transfer coefficient solution. The local HCOEF distribution results at $\mathrm{Re}=7,000$ has range between 262-600. At $\mathrm{Re}=14,000$ the HCOEF range is $100-1040$. As expected the highest HCOEF is at $\mathrm{Re}=21,000$ with a range of 183-1400.

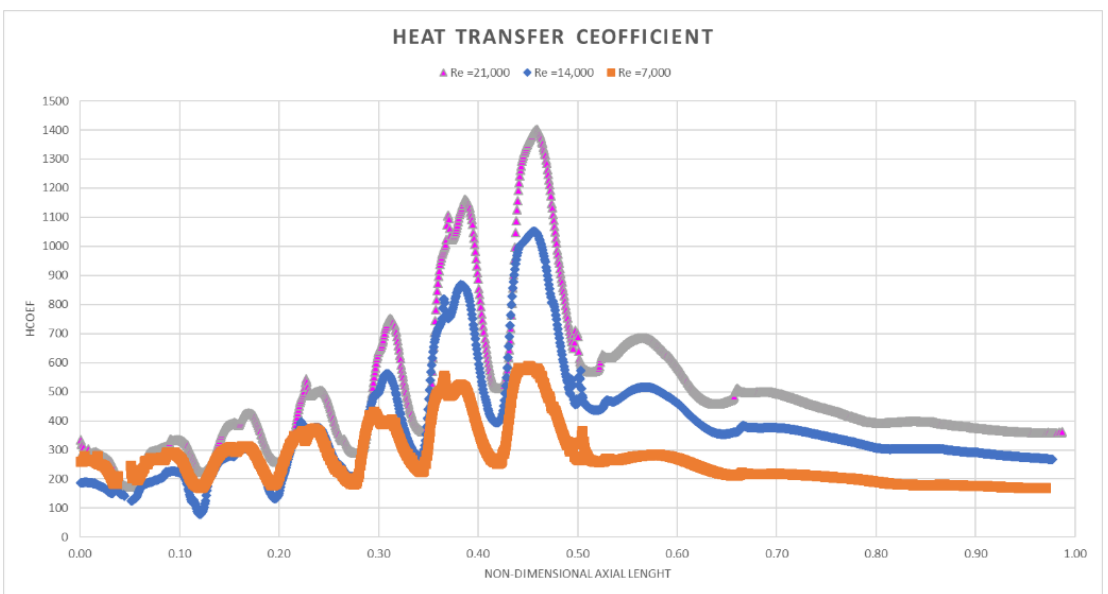

Fig. 10: Heat Transfer Coefficient at $\operatorname{Re}=7,000,14,000$, and 21,000.

The Nusselt number increases as the Re number increases, as shown in Fig. 11. Results indicate that complex Nusselt numbers variations are evident in Fig. 11, specially just downstream of each air inlets located axial length 0.25-0.50. The Nusselt numbers begin to vary near each air inlet in both axial and circumferential directions. The local Nusselt number is based on a transient heat transfer solution of a semi-infinite medium suddenly exposed to a convective boundary condition. At $\mathrm{Re}=14,000$, the experiment indicates an average Nusselt number is higher in the middle of the chamber length when comparing results to Hedlund et al. [12] . One explanation is that the current test article has seven air inlets versus Hedlund's test article of only two air inlets. For $\mathrm{Re}=7,000$ local Nusselt numbers from axial length 0.15 to 0.5 fluctuate along the entire interior concave surface of the swirl chamber, Nusselt number range is 180-600. The distribution of Nusselt number at Re $=14,000$, with a range of 90-1000, occurs at a higher rate downstream the chamber when compared to the upstream. The Nu results at $\operatorname{Re}=21,000$ has a range of 200-1350, with higher peak at the middle of the chamber.

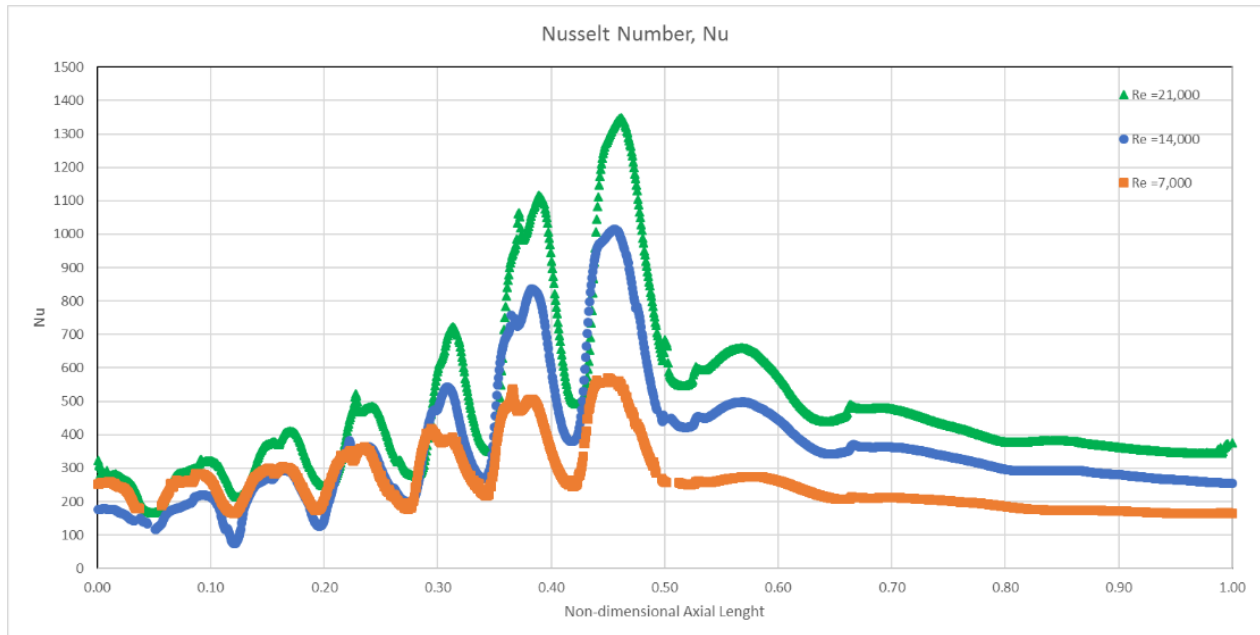

Fig. 11: Nusselt Number at $\operatorname{Re}=7,000,14,000$, and 21,000. 


\section{Conclusion}

In conclusion heat transfer was augmented by tangential impingement effects, swirl motions, and high velocity vortices formed at the near wall region. The present geometry, which has been investigated at the early stage of the swirl cooling techniques development, provides valuable information to understand the effects of tangential air inlets' leading edge diameter, leading-edge length, and flow rates on the heat transfer augmentation. The seven discrete tangential air inlets along the circular chamber axis allows the optimization between the heat transfer augmentation and manufacturability.

The circumferential velocity decreases and maximum circumferential velocity shifts towards the center of the swirl chamber with downstream distance. At the outer wall region, the axial velocity is higher and increases within the chamber length. After the second half of the chamber length the axial velocity reaches its maximum. It is further observed that the magnitude of the vorticity increases closer to the wall and downstream chamber length. The swirl increases are visible from air inlet number two through seven and later swirl number decreases as is predicted, since there are no more air inlets. Also, flow near the inlets are augmented swirl numbers produced by the shear layer near the edge of the air inlet. At $\operatorname{Re}=21,000$, the air inlet number 3 measured the maximum swirl number which of 3.3. This implies that there is a high flux circumferential momentum relative to flux axial momentum. Results show that the more air inlets equated to higher Swirl and Nusselt numbers at each of the air inlets. The highest heat transfer coefficients are found in the mid passage of the circular. The heat transfer measurements on the circular chamber model showed a higher internal heat transfer coefficient than predicted. The 3-D results will help to achieve improved cooling designs in the challenging regions to maximize the heat transfer in the internal wall region of the turbine leading edge. Further CFD modelling investigations are needed for internal cooling heat transfer modeling.

\section{Acknowledgements}

The work presented in this paper was performed at Solar Turbines. Geoffrey Potts, Kurt Mueller, Robert Corey Hoffman, Mike Austin, and Michael D. Wolver are acknowledged for many valuable discussions on the research.

\section{Nomenclature}

D circular chamber diameter, $m$

$\mathrm{D}_{\mathrm{H}} \quad$ hydraulic diameter of one swirl chamber inlet

$\mathrm{H}$ height

$h \quad$ heat transfer coefficient, $\mathrm{W} /\left(\mathrm{m}^{2} \mathrm{~K}\right)$

$\mathrm{k}$ turbulent kinetic energy, $\mathrm{m}^{2} / \mathrm{s}^{2}$

K thermal conductivity

L circular chamber length, $\mathrm{m}$

m mass flow rate, $\mathrm{kg} / \mathrm{s}$

$\mathrm{R}$ radial distance measured from chamber centerline

Re Reynolds number

$\mathrm{r}$ radial distance, $\mathrm{m}$

$\mathrm{r}, \phi, \mathrm{z}$ cylindrical coordinates

$\mathrm{S}_{\mathrm{N}} \quad$ swirl number

$t \quad$ temperature, ${ }^{\circ} \mathrm{C}$

$\mathrm{V}_{\mathrm{z}} \quad$ axial velocity

$\mathrm{V}_{\phi} \quad$ circumferential velocity

W air inlet width
Greek symbols

$\begin{array}{ll}\rho & \text { density } \\ \mu & \text { absolute viscosity } \\ \nu & \text { kinematic viscosity } \\ \omega_{Z} & \text { circumferential component of } \\ & \text { vorticity } \\ \text { ipts and superscripts } \\ H & \text { hydraulic } \\ Z & \text { axial } \\ \phi & \text { circumferential }\end{array}$

\section{References}

[1] H.-K. Moon, T. O'Connell, and B.Glezer, "Heat Transfer Enhancement in a Circular Channel Using Lenghtwise Continuous Tangential Injection," Heat Transfer, vol. 6, pp. 559-564, 1998.

[2] B. M. H.-K. K. J. B. J. a. G. G. Glezer, "Heat Transfer in a Rotating Radial Channel with Swirling Internal Flow," ASME, pp. 1-7, 1998. 
[3] P. M. Ligrani, C. R. Hedlund, R. Thambu, B. T. Babinchak, H.-K. Moon and B. Glezer, "Flow Phenomena in Swirl Chambers," The American Society of Mechanical Engineers, 1997.

[4] F. Kreith and D. Margolis, "Heat Transfer and Friction in Turbulent Vortex Flow," Applied Science, vol. 8, no. A, pp. $1-17$.

[5] C. Camci, and B. Glezer, "Color Response Modification of Encapsulated Liquid Crystals Used in Rotating Disk Heat Transfer Studies," ASME, pp. 1-10, 1995.

[6] F. Chang, and V. Dhir, "Mechanisms of Heat Transfer Enhancement and Slow Decay of Swirl in Tubes Using Tangential Injection," Heat and Fluid Flow, vol. 16, pp. 78-87, 1995.

[7] B. Glezer, H. K. Moon, and T. O'Connell, “A Novel Technique For The Internal Blade Cooling," The American Society of Mechanical Engineers, pp. 1-10, 1996.

[8] D. Galeana, and A. Beyene, "Experimentatl Investigation of Heat Transfer and Swirl Cooling Flow in a Circular Chamber Using Liquid Crystals and 3-D Stereo-PIV," in Efficiency, Cost, Optimization, Simulation and Enviromental Impact of Energy Systems, Guimaraes, Portugal, 2018.

[9] Carl R. Hedlund, and P. Ligrani, "Local Swirl Chamber Heat Transfer and Flow Structure at Different Reynolds Numbers," Journal of Turbomachinary, vol. 122, pp. 374-385, 2000.

[10] D. Galeana, and A. Beyene, "Experimental Study of Swirl Cooling Flow on a Circular Chamber Using 3-D StereoPIV," in Power \& Energy Conference \& Exhibition ASME, Lake Buena Vista, FL, USA, 2018.

[11] B. Wieneke, "Generic a-posteriori uncertainty quantification for PIV vector fields by correlation statistics," 17th International Symposium on Applications of Laser Techniques to Fluid Mechanics, pp. 1-9, 2014.

[12] C. L. P. M. H.-K. a. G. B. Hedlund, "Heat Transfer and Flow Phenomena in a Swirl Chamber Simulating Turbine Blade Internal Cooling," ASME, vol. 121, pp. 804-813, 1999.

[13] B. Glezer, H.-K. Moon, and T. O'Connell, "A Novel Technique For The Internal Blade Cooling," The American Society of Mechanical Engineers, pp. 1-10, 1996.

[14] P. M. Ligrani, M. M. Oliveira, and T. Blaskovich, "Comparison of Heat Transfer Augmentation Techniques," AIAA Journal, vol. 41, no. 3, pp. 1-26, 2003.

[15] R. Thambu, T. Babinchak, P. Ligrani, C. R. Hedlund, H.-K. Moon, and B. Glezer, "Flow in a Simple Swirl Chamber With and Without Controlled Inlet Forcing," Experiments in Fluids, vol. 26, pp. 347-357, 1999.

[16] C. R. Hedlund and P. M. Ligrani, "Local Swirl Chamber Heat Transfer and Flow Structure at Different Reynolds Numbers," The American Society of Mechanical Engineers, pp. 1-12, 30 September 1999. 